\title{
Histone deacetylase 4 mediates SMAD family member 4 deacetylation and induces 5-fluorouracil resistance in breast cancer cells
}

\author{
SEONG-LAN YU ${ }^{1 *}$, DONG CHUL LEE ${ }^{2 *}, \mathrm{JI}_{\text {WOONG SON }}^{3}$, CHANG GYO PARK $^{1}$, \\ HOI YOUNG LEE ${ }^{1}$ and JAEKU KANG ${ }^{1}$ \\ ${ }^{1}$ Department of Pharmacology, College of Medicine, Konyang University, Daejeon 302-718; \\ ${ }^{2}$ Medical Genomics Research Center, Korea Research Institute of Bioscience and Biotechnology, \\ Daejeon 305-806; ${ }^{3}$ Department of Internal Medicine, Konyang University Hospital, \\ Daejeon 302-718, Republic of Korea
}

Received May 1, 2013; Accepted June 12, 2013

DOI: $10.3892 /$ or.2013.2578

\begin{abstract}
Histone deacetylases (HDACs) have been shown to play important roles in the regulation of chromatin remodeling by histone deacetylation, and their expression is induced in several types of cancer. In addition, they are known to be associated with resistance to anticancer drugs. However, the relevance of HDAC4 in chemoresistance remains unclear. Therefore, we investigated the interaction between HDAC4 expression and chemoresistance in breast cancer cells. We found that increased HDAC4 expression in MDA-MB-231 cells was associated with resistance to the anticancer drug 5-fluorouracil (5-FU). To verify these results, a cell line stably overexpressing HDAC4 was generated using MCF-7 cells (HDAC4OE). This cell line displayed increased 5-FU resistance, and HDAC4 knockdown in HDAC4OE cells restored 5-FU sensitivity. Consequently, we concluded that HDAC4 is a critical gene associated with 5-FU chemoresistance. Further investigation using a microarray approach revealed that 355 genes were differentially expressed following HDAC4 overexpression. Based on functional annotation of the array results, HDAC4 overexpression was found to downregulate genes related to the transforming growth factor (TGF) $\beta$ signaling pathway, including SMAD4, SMAD6, bone morphogenetic protein 6 , inhibitor of DNA binding 1 and TGF 32 . We also found that HDAC4 expression regulates SMAD4 expression by inducing deacetylation of histone $\mathrm{H} 3$ in the SMAD4 promoter region. In addition, SMAD4 knockdown in MCF-7
\end{abstract}

Correspondence to: Dr Jaeku Kang, Department of Pharmacology, College of Medicine, Konyang University, Daejeon 302-718, Republic of Korea

E-mail: jaeku@konyang.ac.kr

*Contributed equally

Key words: histone deacetylase 4, SMAD family member 4, 5-fluorouracil, chemoresistance, breast cancer cells cells increased 5-FU resistance. In summary, our data suggest that HDAC4-mediated deacetylation of the SMAD4 promoter may lead to 5-FU resistance in breast cancer cells.

\section{Introduction}

Numerous types of cancer develop as a result of dysregulated gene expression derived from epigenetic modifications such as methylation or acetylation $(1,2)$. In particular, DNA-bound core histones regulate gene activation and inactivation via the opposing activities of histone acetyltransferase (HAT) and histone deacetylase (HDAC) enzymes.

HDACs play crucial roles in epigenetic modulation by deacetylating histone and non-histone substrates that are involved in processes critical for both normal development and cancer (3). HDACs have been classified into four classes based on structural homologies between humans and yeast. Class I HDACs are ubiquitously expressed in the nuclei of all cells (4), whereas HDACs 4, 5, 7 and 9, which are members of class II, are shuttled between the nucleus and the cytoplasm and are specifically expressed in heart tissue, bone, the nervous system and skeletal muscle (5). In several types of cancer, aberrant expression of HDAC family members has been proposed as a hallmark of multiple tumorigenic processes, including proliferation, apoptosis, angiogenesis and metastasis (6). Moreover, a number of researchers have suggested that HDACs are associated with chemotherapy resistance in several types of cancer (7-11). Chemoresistance to effective treatments for many tumor types presents a major obstacle, leading researchers to study the use of molecularly targeted therapies as a new class of chemotherapeutic agents. HDAC inhibitors, which target HDAC proteins, have a wide range of effects, including cell cycle arrest, apoptosis, anti-angiogenic effects, and autophagy, and are utilized clinically as chemotherapeutic agents in several types of cancer (5).

We previously reported that HDAC expression in metastatic and non-metastatic breast cancer cells differs and that high HDAC expression levels, with the exception of HDAC4, are associated with invasiveness, which is of concern due to 
the role in invasion of cancer metastasis (12). Furthermore, Stronach et al (13) recently demonstrated that HDAC4 may be a therapeutic target for platinum resistance in ovarian cancer.

SMAD family member 4 (SMAD4) has also been reported to be a gene that promotes 5-fluorouracil (5-FU) resistance in colon cancer (14). SMAD4 is known to be a mediator of the transforming growth factor (TGF) $\beta$ signaling pathway and has been shown to act as a suppressor of tumor progression and to decrease tumor growth by inducing apoptosis. SMAD4 inactivation by genetic abnormalities, including deletion or mutation, has been identified in several types of cancer, including colorectal, pancreatic, breast, ovarian, lung and gastric cancer (15-19). Furthermore, reduction of SMAD4 expression by methylation of the SMAD4 promoter has been correlated with advanced prostate cancer (20). However, the correlation between HDAC4 and SMAD4 has not been elucidated.

In the present study, we investigated the correlation between high HDAC4 expression and chemoresistance as well as the molecular targets that are associated with HDAC4 overexpression-induced chemoresistance in breast cancer. Based on these results, we suggest that SMAD4 in the TGF $\beta$ signaling pathway is regulated by HDAC4 and is associated with 5-FU resistance in breast cancer cells.

\section{Materials and methods}

Cell culture and transfection. MDA-MB-231 (HTB-26) and MCF-7 (HTB-22) breast cancer cells that had been obtained from the American Type Culture Collection (ATCC) were cultured in Dulbecco's Modified Eagle's Medium (DMEM; Lonza) supplemented with $10 \%$ fetal bovine serum (FBS; Gibco) and $1 \%$ penicillin/streptomycin (Lonza). The cells were maintained at $37^{\circ} \mathrm{C}$ in a humidified atmosphere containing $5 \% \mathrm{CO}_{2}$.

To obtain stable expression of the HDAC4 protein, MCF-7 cells were transfected with Lipofectamine ${ }^{\circledR} 2000$ (Invitrogen) using a human HDAC4 full-length cDNA vector constructed in a pcDNA3-EGFP Plasmid (Addgene) carrying the neomycin resistance gene. Transfected clones were cultured in selective media supplemented with $600 \mu \mathrm{g} / \mathrm{ml}$ of G418 (Sigma).

Transient transfection was performed using Lipofectamine ${ }^{\circledR}$ 2000 in order to overexpress hHDAC4 and the Lipofectamine ${ }^{\circledR}$ RNAiMAX Reagent (Invitrogen) was used to knockdown hHDAC4 and hSMAD4 expression according to the manufacturer's recommended protocol.

Quantitative real-time PCR ( $q R T$-PCR). Total RNA was extracted using a Nucleospin RNAII kit (Macherey-Nagel, Germany) and cDNAs were synthesized using M-MLV Reverse Transcriptase (Promega). qRT-PCR reactions were carried out in triplicate using $\mathrm{iQ}^{\mathrm{TM}}$ SYBR-Green Supermix and a CFX96 qPCR machine (BioRad). The primers used to detect HDAC4, SMAD4 and glyceraldehyde-3-phosphate dehydrogenase (GAPDH) were: HDAC4 forward, 5'-agcgtgagcaagatcctc-3' and reverse, 5'-gccaagtactcagcgtctcc-3'; SMAD4 forward, 5'-cccaggatcagtaggtggaa-3' and reverse, 5'-cccagctttcacaaaactc-3'; and GAPDH forward, 5'-acagtcagccgcatcttctt-3' and reverse, 5'-acgaccaaatccgttgactc-3'. The amplification conditions were: a predenaturation step at $95^{\circ} \mathrm{C}$ for $3 \mathrm{~min}$, followed by 40 cycles of denaturation at $95^{\circ} \mathrm{C}$ for $15 \mathrm{sec}$, annealing at $60^{\circ} \mathrm{C}$ for $15 \mathrm{sec}$ and extension at $72^{\circ} \mathrm{C}$ for
15 sec. The comparative threshold cycle $(\mathrm{Ct})$ method, $2^{-\Delta \Delta \mathrm{Ct}}$, was used to calculate fold amplification.

Western blot analysis. The cells were lysed with RIPA buffer containing $50 \mathrm{mM}$ Tris- $\mathrm{HCl}$ at $\mathrm{pH} \mathrm{7.4,1 \%} \mathrm{NP-40,0.25 \%}$ sodium deoxycholate, $150 \mathrm{mM} \mathrm{NaCl}, 1 \mathrm{mM}$ EDTA, $1 \mathrm{mM}$ $\mathrm{Na}_{3} \mathrm{Vo}_{4}, 1 \mathrm{mM} \mathrm{NaF}$ and proteinase inhibitors. Proteins were separated using $8 \%$ SDS-PAGE gels and transferred to polyvinylidene difluoride membranes (PVDF; Millipore, Germany). Blots were then incubated in 5\% skim milk (Difco) for $1 \mathrm{~h}$ and probed with anti-HDAC4 (Abcam) and anti-GAPDH (Santa Cruz Biotechnology, Inc.) primary antibodies overnight, followed by incubation with a horseradish peroxidase (HRP)-conjugated anti-rabbit secondary antibody. Immunoreactive proteins were detected using an enhanced chemiluminescence kit (Thermo).

In vitro cytotoxicity assay. To identify differences in the cytotoxicity of anticancer drugs, including cisplatin (Sigma), paclitaxel (Bristol-Myers Squibb SRL, Italy), gemcitabine (Lilly, Korea) and 5-FU (Sigma), cells were plated in 96-well plates at a density of $5 \times 10^{3}$ cells/well. Twenty-four hours later, the cells were treated with anticancer drugs and incubated for 24, 48 and $72 \mathrm{~h}$. The surviving cells were treated with $500 \mu \mathrm{g} / \mathrm{ml}$ of MTT solution for $2 \mathrm{~h}$, after which point the absorbance was measured at $540 \mathrm{~nm}$. The survival rate was calculated as the ratio of the absorbance of the treated wells to that of the control wells.

Microarray analysis. Biotinylated cRNA was produced according to the manufacturer's protocol. Briefly, total RNA was reverse-transcribed to cDNA using T7 oligo(dT) primers and second-strand cDNAs were synthesized through in vitro transcription and labeled with biotin-NTP. The amounts of the labeled cDNAs were quantified using an ND-1000 Spectrophotometer (NanoDrop).

Labeled cRNA samples were hybridized to each human HT-12 v4 Expression Bead Array (Illumina, Inc.) for $16-18 \mathrm{~h}$ at $58^{\circ} \mathrm{C}$. Amersham fluorolink streptavidin-Cy3 (GE Healthcare Bio-Sciences, UK) and an Illumina Bead Array Reader confocal scanner were used to detect the array signal according to the manufacturer's instructions. The microarray experiments on independent samples were performed in four times replicate.

For data analysis, raw data were extracted from the software provided by the manufacturer (GenomeStudio v2011.1; Illumina, Inc.). The raw data were then filtered using a detection P-value of $<0.05$ in $\geq 50 \%$ of the samples. Selected gene signal values were transformed and normalized using the logarithmic and quantile methods, respectively. Significant statistical values for the expression data were determined using fold-change and the local pooled error (LPE) test. The false discovery rate (FDR) was controlled by adjusting the P-values using the Benjamini-Hochberg algorithm.

Functional annotation analysis of the significant probe list was performed using the Database for Annotation, Visualization and Integrated Discovery (DAVID) (http://david. abcc.ncifcrf.gov/home.jsp).

The data discussed in this publication have been deposited in the NCBI gene expression omnibus (GEO) and are accessible 

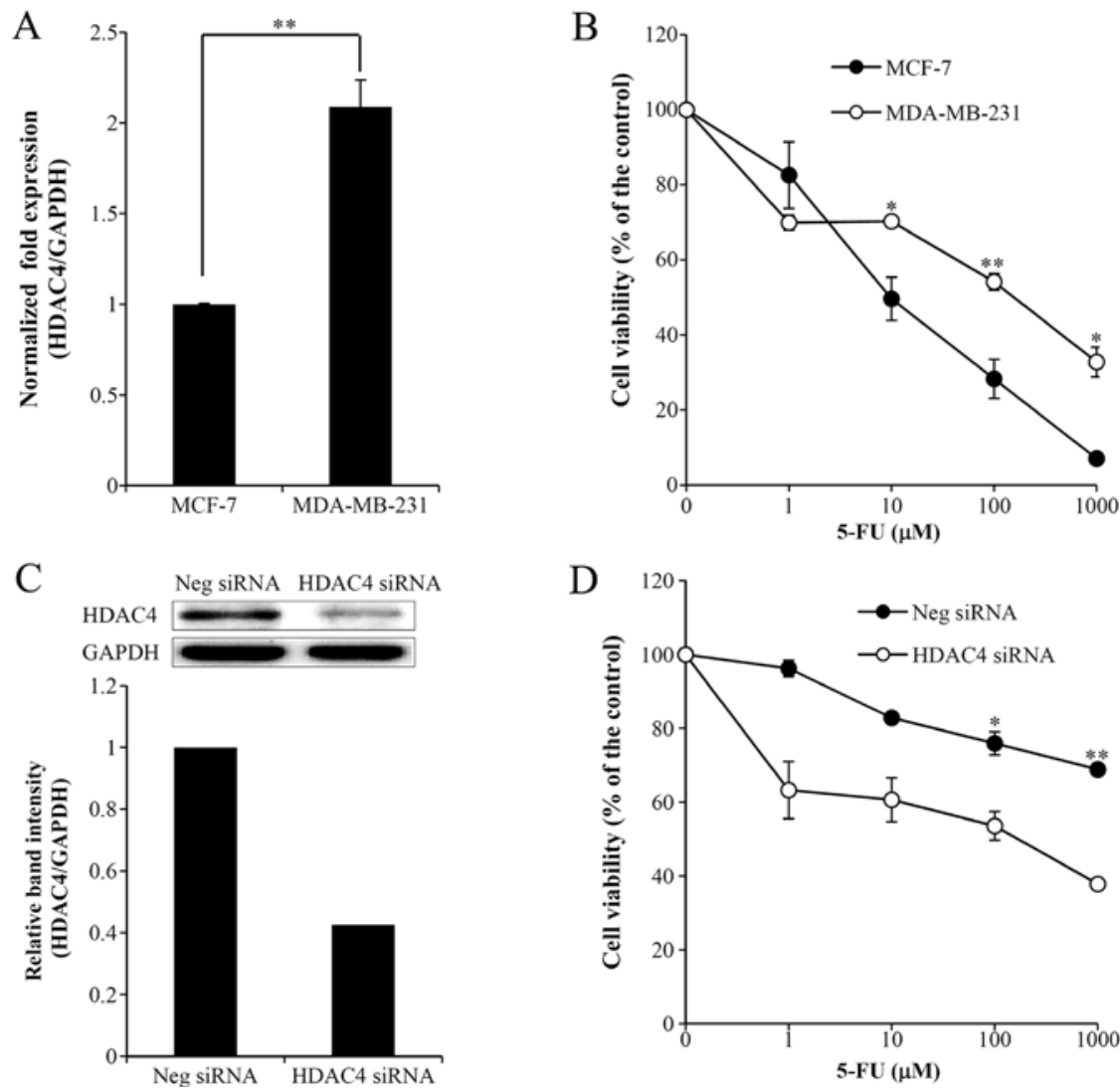

Figure 1. Histone deacetylase (HDAC) 4 expression affects 5-fluorouracil (5-FU) resistance in breast cancer cells. (A) Quantitative real-time PCR analysis of HDAC4 expression in MDA-MB-231 and MCF-7 cells. (B) Viability of MDA-MB-231 and MCF-7 cells after 5-FU treatment at different dosages for $72 \mathrm{~h}$. (C) Western blot analysis of HDAC4 knockdown in MDA-MB-231 cells. (D) HDAC4 knockdown significantly decreased 5-FU cytotoxicity in MDA-MB-231 cells. The bars and each point on the graphs represent the means \pm SD of three independent experiments. ${ }^{* *} \mathrm{P}<0.01$ and ${ }^{*} \mathrm{P}<0.05$ as compared to the control, analyzed using the Student's t test.

through the GEO series accession number GSE42242 (http:// www.ncbi.nlm.nih.gov/geo/query/acc.cgi?acc=GSE42242).

Chromatin immunoprecipitation (ChIP) assay. ChIP assays were performed using a ChIP Assay kit (Millipore) according to the manufacturer's protocol. Briefly, cells were cross-linked with $1 \%$ formaldehyde and sonicated on ice in order to fragment the chromatin. The lysates were diluted with chromatin-dilution buffer and the soluble fractions were immunoprecipitated using anti-acetyl-histone H3 (Millipore) at $4^{\circ} \mathrm{C}$ overnight. Protein-A Sepharose beads were complexed with anti-acetyl-histone $\mathrm{H} 3$ and washed to remove nonspecific binding. The antibody-bound chromatin was eluted using elution buffer ( $1 \%$ SDS and $0.1 \mathrm{M} \mathrm{NaHCO}_{3}$ ). The eluted chromatin samples were treated with proteinase $\mathrm{K}$ at $45^{\circ} \mathrm{C}$ for $1 \mathrm{~h}$ and the DNA was extracted using the phenol chloroform method. Finally, PCR was performed on the eluted DNA using four primer sets that were targeted to the SMAD4 promoter region. The sequences of the primers targeting the SMAD4 promoter were: P1 forward, 5'-gtggaaggaggagcagtgtc-3' and reverse, 5'-tgtcacctttgccatacattg-3'; P2 forward, 5'-tgtgtgtttccttcccettc-3' and reverse, 5'-tccttgcaggctacaggact-3'; P3 forward, 5'-tcctttgttccagcetcact-3' and reverse, 5'-aaa ctgaaggaagatctgtcagc-3'; P4 forward, 5'-tgaaattacceggatgt ggt-3' and reverse, 5'-ctaggggagagcaggaagg-3'; and P5 forward, $5^{\prime}$-gctcgtgggagaatcaagtt- 3 ' and reverse, $5^{\prime}$-caaaacagaaattgg ctgga-3'. The PCR products were analyzed on $2 \%$ standard
Tris/Acetate/EDTA (TAE) agarose gels that had been stained with GelRed ${ }^{\mathrm{TM}}$ Nucleic Acid Gel Staining solution (Biotium).

Statistical analyses. All graphed data are presented as the means \pm standard deviation (SD). The results were analyzed using analysis of variance (ANOVA) and the Student's t-test. P-values $<0.05$ or 0.01 were considered to indicate statistically significant differences.

\section{Results}

HDAC4 expression affects 5-FU chemoresistance in breast cancer cells. We previously reported that HDAC expression was associated with cancer progression in metastatic MDA-MB-231 and non-metastatic MCF-7 cells. As a result, the increased expression of HDAC1, 6 and 8 in MDA-MB-231 cells as compared to MCF-7 cells was correlated with tumor cell invasiveness. Although the expression of HDAC4 was increased in MDA-MB-231 cells, HDAC4 did not increase tumor cell invasion (12). To investigate whether increased HDAC4 expression may be associated with chemoresistance to anticancer drugs, we verified the cytotoxicity of various anticancer drugs, including cisplatin, paclitaxel, gemcitabine and 5-FU, in MCF-7 and MDA-MB-231 cells (data not shown). Significant chemoresistance was demonstrated only for 5-FU. As shown in Fig. 1B, more pronounced 5-FU chemoresistance was observed in MDA-MB-231 cells than in MCF-7 
A

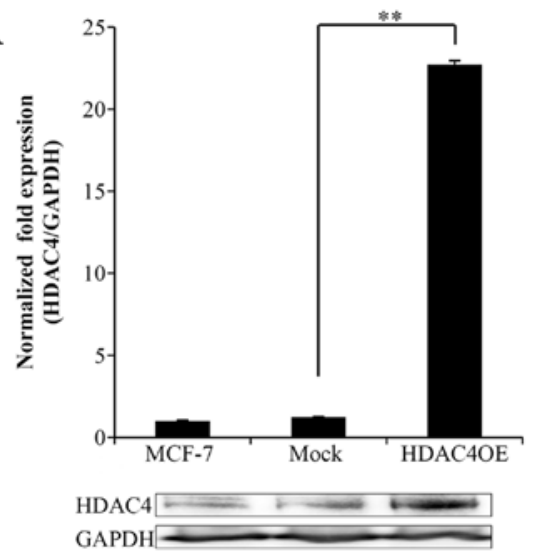

$\mathrm{C}$

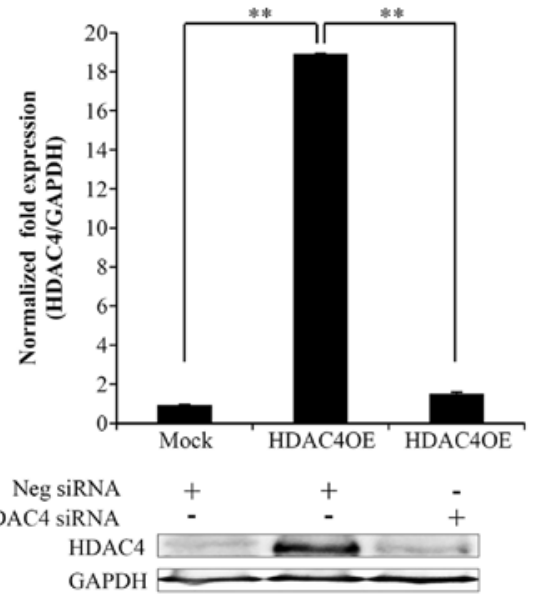

B

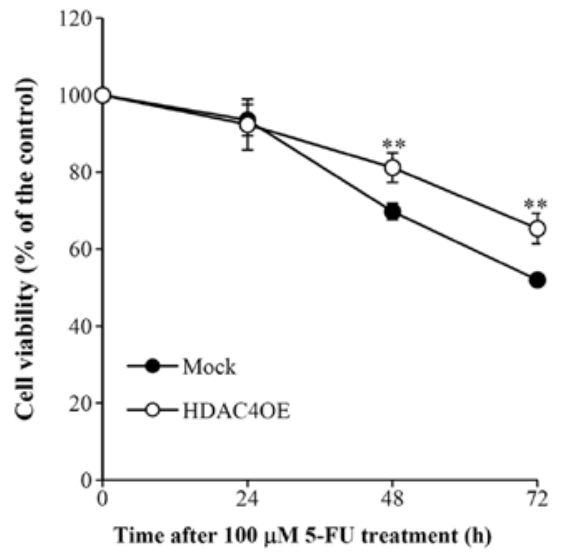

$\mathrm{D}$

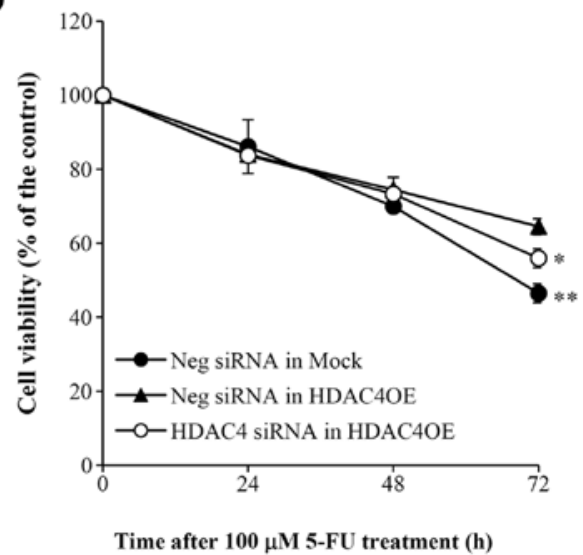

Figure 2. 5-Fluorouracil (5-FU) chemoresistance in MCF-7 cells is dependent on histone deacetylase (HDAC) 4 expression levels. (A) Quantitative real-time PCR (qRT-PCR; upper panel) and western blot (lower panel) analyses of HDAC4 expression in mock-transfected and HDAC4OE cells. (B) The viability of HDAC4OE cells increased after exposure to $100 \mu \mathrm{M}$ 5-FU. (C) qRT-PCR (upper panel) and western blot (lower panel) analyses of HDAC4 knockdown in mock-transfected and HDAC4OE cells. (D) HDAC4 knockdown in HDAC4OE cells rescued cell viability following treatment with 100 $\mu \mathrm{M}$ 5-FU. Data are represented as the means \pm SD of three independent experiments. ${ }^{* *} \mathrm{P}<0.01$ and ${ }^{*} \mathrm{P}<0.05$ as compared to the control, analyzed using the Student's t-test.

cells, confirming that the decreased HDAC4 expression in MDA-MB-231 cells transfected with siRNA against HDAC4 was associated with reduced 5-FU resistance (Fig. 1C and D). Therefore, we proposed that HDAC4 expression may be associated with 5-FU chemoresistance. To test the direct relationship between HDAC4 and 5-FU resistance, we established MCF-7 cells stably overexpressing HDAC4 (HDAC4OE). MCF-7 cells with elevated HDAC4 expression were shown to be more resistant to 5-FU than control cells (Fig. 2A and B). Moreover, we found that HDAC4 knockdown with siRNA in HDAC4OE cells reversed 5-FU chemoresistance (Fig. 2C and D). These observations suggested that HDAC4 participates in 5-FU chemoresistance.

Identification of significant HDAC4 overexpression-mediated alterations in the TGF $\beta$ signaling pathway in MCF-7 cells. To identify differentially expressed genes in HDAC4OE cells, we performed microarray analysis in mock-transfected and HDAC4OE cells. Among the genes regulated by HDAC4 overexpression, 355 genes were shown to have significantly different expression levels (the expression of 185 genes was increased, and the expression of 170 genes was decreased) based on the criteria of a 1.5 -fold difference with a $\mathrm{P}$-value $<0.05$. Hierarchical clustering analysis was performed using differentially expressed genes (Fig. 3A). To investigate alternative pathways of differentially expressed genes, the functional gene ontology of each gene was analyzed against the DAVID. As a result, 285 genes were defined (Fig. 3B). We identified 84 genes, including SMAD4, SMAD6, bone morphogenetic protein 6 (BMP6), inhibitor of DNA binding 1 (ID1), TGF 32 and TGF $\beta 3$, involved in the TGF $\beta$ signaling pathway from the most significant cluster $(\mathrm{P}<0.01)$ (Fig. 3C and D). Recently, Papageorgis et al (14) reported that SMAD4 inactivation induced 5-FU resistance in colon cancer. Therefore, we selected SMAD4 as a candidate gene that may regulate 5-FU chemoresistance in HDAC4OE cells.

HDAC4-mediatedregulation of the SMAD4 gene impacts 5-FU chemoresistance. To confirm the correlation between SMAD4 and HDAC4, the pattern of SMAD4 expression in HDAC4OE and MDA-MB-231 cells was characterized using qRT-PCR. SMAD4 expression was downregulated in cells in which HDAC4 was highly expressed (Fig. 4A), and SMAD4 expression was upregulated when HDAC4 siRNA was transduced into HDAC4OE cells (Fig. 4B). Consequently, we hypothesized that HDAC4 may regulate SMAD4 expression in breast cancer cells. To investigate alterations in chemoresistance mediated by SMAD4 expression due to HDAC4 overexpression in MCF-7 cells, we performed cytotoxicity assays comparing SMAD4 knockdown and control MCF-7 cells. As a result, the 
A

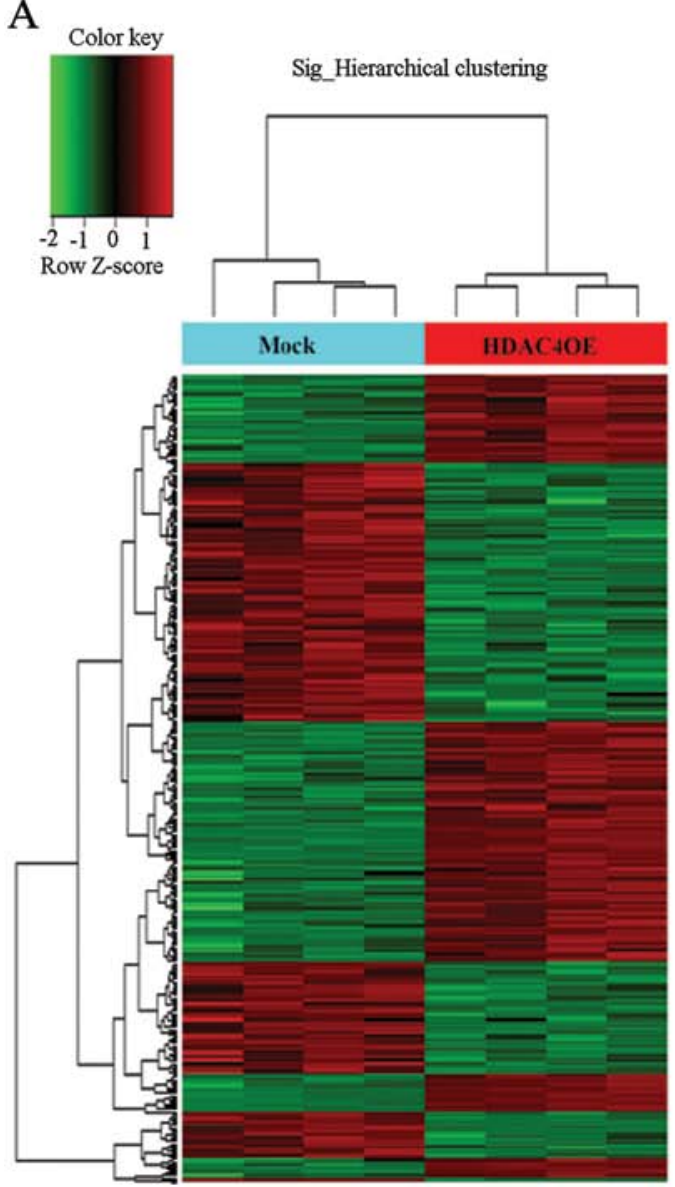

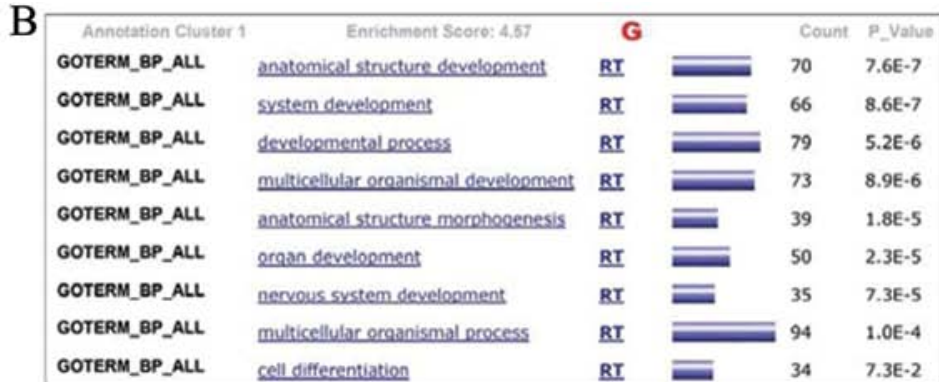

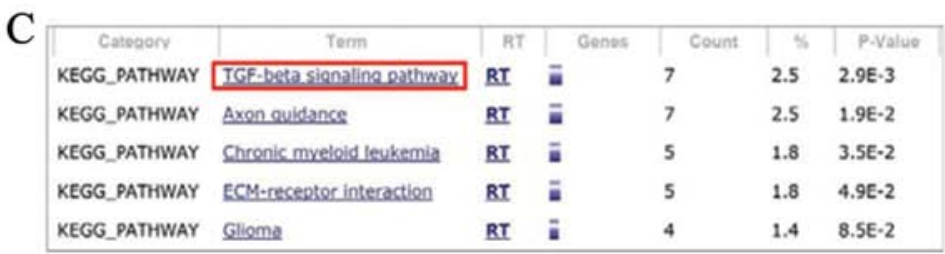

$\mathrm{D}$

\begin{tabular}{|c|c|c|c|c|}
\hline Gene Name & Gene symbol & GenBank & Log2Ratio & p value \\
\hline SMAD family , & SMAD4 & NM_005359.3 & -1.6178 & 1.31E-09 \\
\hline SMAD family member 6 & SMAD6 & NM_005585.2 & -1.6149 & $3.81 \mathrm{E}-0$ \\
\hline bone morphogenetic protein 6 & BMP6 & NM_001718.4 & -1.5367 & 2.95E-06 \\
\hline cyclin-dependent kinase inhibitor $2 \mathrm{~B}$ & CDKN2B & NM_004936.3 & -2.1321 & $5.83 \mathrm{E}-22$ \\
\hline inhibitor of DNA binding 1 & IDI & NM_181353.1 & -1.5646 & 9.65E-1 \\
\hline transforming grow & TGFB2 & NM_003238.1 & -3.0364 & $8.48 \mathrm{E}-45$ \\
\hline transforming growth factor, beta 3 & TGFB3 & NM_003239.I & 1.5416 & $1.14 \mathrm{E}-0$ \\
\hline
\end{tabular}

Figure 3. Identification of significant histone deacetylase (HDAC) 4 overexpression-mediated alterations in the transforming growth factor (TGF) $\beta$ signaling pathway in MCF-7 cells using microarray. (A) Hierarchical clustering of significant differences in gene expression between mock-transfected and HDAC4OE cells. (B) Gene ontology analysis (GOTERM_BP_ALL) was carried out using functional annotation clustering against the DAVID online tool. (C) Significant alterations in the TGF $\beta$ pathway were identified in HDAC4OE cells using pathway analysis (KEGG_PATHWAY) against the DAVID online tool. (D) Summary of differentially expressed genes associated with the TGF $\beta$ pathway, as indicated by the results of the microarray analysis.

cytotoxicity of 5-FU in SMAD4 knockdown cells was elevated (Fig. 4C and D). Therefore, SMAD4 can be reliably associated with 5-FU resistance in breast cancer cells.

HDAC4 regulates SMAD4 gene expression via deacetylation of the SMAD4 promoter. To determine whether SMAD4 transcriptional repression by HDAC4 is mediated through a modification of histones in the SAMD4 promoter region, we compared the SMAD4 promoter region to the UCSC genome browser (http://genome.ucsc.edu/) and found that histone acetylation was enriched. We also identified a putative binding site for yin-yang 1 (YY1), which is known to transcriptionally repress several genes by recruiting HDAC4 (21-24) (Fig. 5A). The acetylation status of histones in defined regions of SMAD4 was investigated using ChIP assays comparing acetylation between control and HDAC4OE cells. The ChIP results clearly confirmed that acetylation of histone $\mathrm{H} 3$ was reduced at five regions within the SMAD4 promoter following overexpression of HDAC4 (Fig. 5B). Furthermore, ChIP assays using a HDAC4 antibody were unable to detect the SMAD4 band, which indicated that HDAC4 does not bind directly to the SMAD4 promoter region (data not shown). Consequently, we hypothesized that HDAC4 may form a complex with a transcription factor bound to the SMAD4 promoter.

\section{Discussion}

Alterations in the global pattern of histone acetylation have been observed in several types of cancer. For instance, Fraga et al (25) reported that loss of acetylation at lysine 16 of histone 4 (H4K16) is a common characteristic of cancer cells, and global hypomethylation of lysine 8 of histone 3 (H3K8) has been suggested to be a prognostic indicator of tumorigenesis in breast cancer (26). The relationship between hypomodified and hypermodified histones has also been reported to be associated with mortality in prostate and lung cancer $(27,28)$. In addition, we previously identified differences in HDAC expression between metastatic and non-metastatic breast cancer cells, with high HDAC expression in metastatic breast cancer cells related to invasiveness. However, despite the high expression of HDAC4, it was not shown to participate in invasion (12). Overexpression of HDAC4 has been reported to cause gene-induced resistance to platinum chemotherapy by modulating the acetylation of signal transducer and activator of transcription (STAT) 1 in ovarian cancer (13). In addition, HDAC4 was shown to modulate resistance to docetaxel under hypoxic conditions and the acetylation of HIF1 $\alpha$ in hepatoma cells (9). Therefore, we hypothesized that HDAC4 expression was related to chemoresistance in breast cancer and sought to 

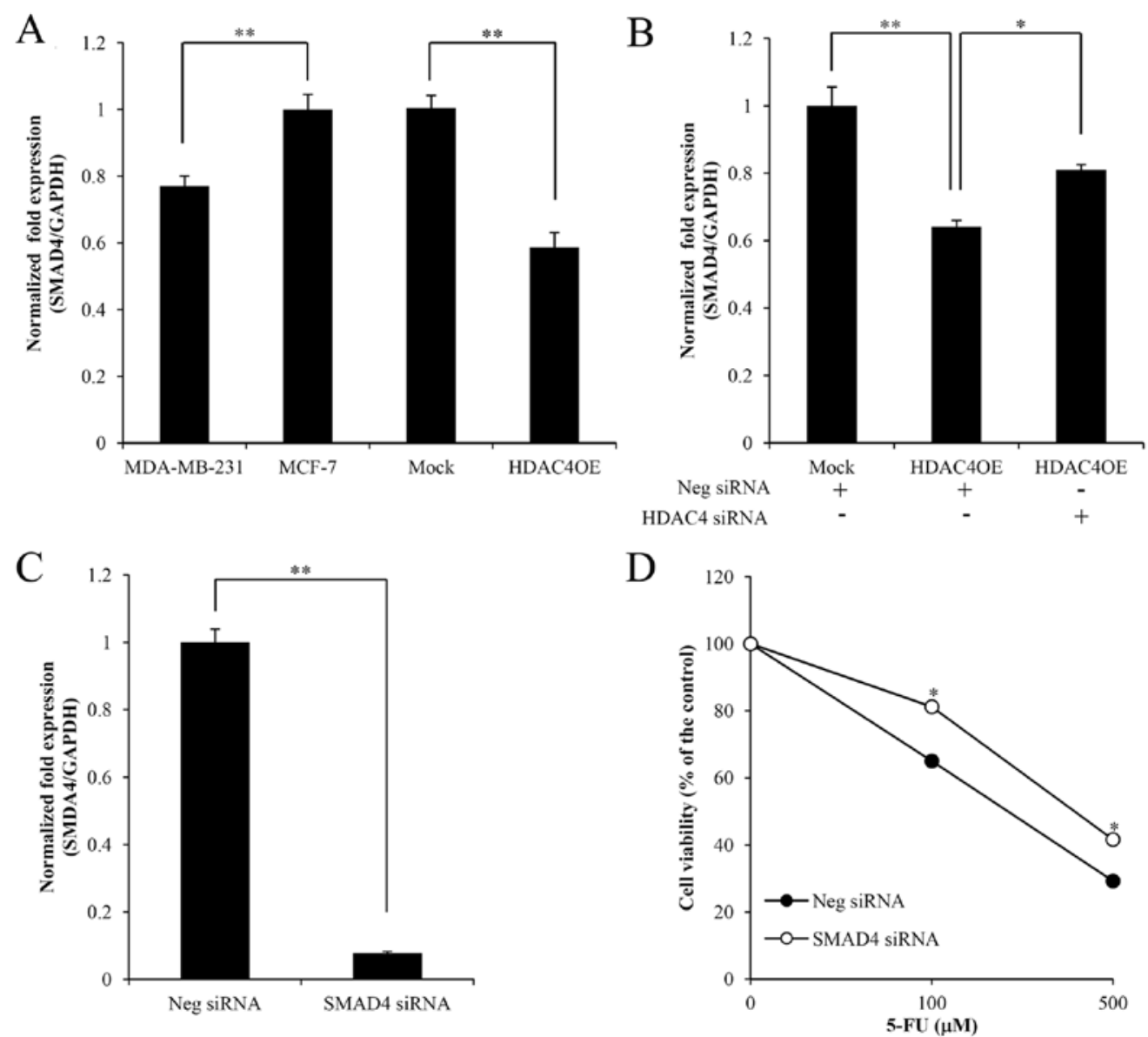

Figure 4. Histone deacetylase (HDAC) 4-mediated SMAD4 downregulation affects 5-fluorouracil (5-FU) resistance in MCF-7 cells. (A) Quantitative real-time PCR (qRT-PCR) analysis of SMAD4 expression in MDA-MB-231, MCF-7, mock-transfected and HDAC4OE cells. (B) SMAD4 expression was increased in HDAC4OE cells that had been transduced with HDAC4 siRNA. (C) qRT-PCR analysis of SMAD4 knockdown in MCF-7 cells. (D) SMAD4 knockdown increased 5-FU resistance. Data represent the means $\pm \mathrm{SD}$ of three independent experiments. ${ }^{* *} \mathrm{P}<0.01$ and ${ }^{*} \mathrm{P}<0.05$ as compared to the control, analyzed using the Student's t test.
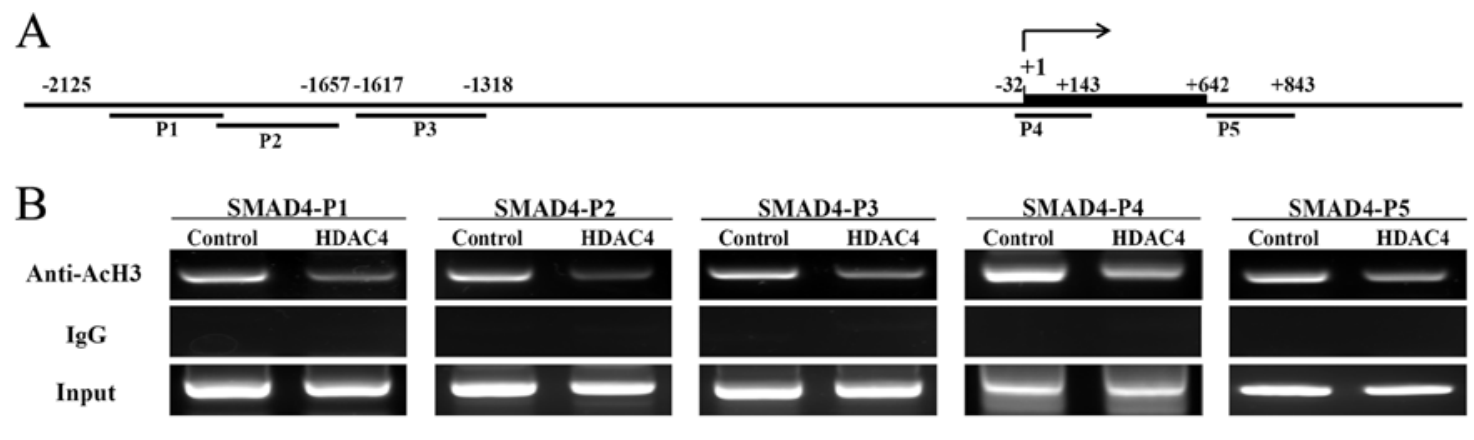

Figure 5. Histone deacetylase (HDAC) 4 alters the acetylation status at the SMAD4 promoter region in MCF-7 cells. (A) Diagram of the 5'-flanking regions of the SMAD4 gene. Primer sets P1-P5 indicate the promoter regions of the SMAD4 gene that were analyzed in chromatin immunoprecipitation (ChIP) assays. (B) ChIP assays were performed in control and HDAC4-overexpressing cells. Immunoprecipitation was performed using an anti-AcH3 antibody.

identify the relationship between HDAC4 expression and 5-FU resistance in breast cancer (Fig. 1B and D). We confirmed that HDAC4 overexpression in MCF-7 cells induced 5-FU resistance, whereas HDAC4 knockdown in HDAC4OE cells restored sensitivity to the drug (Fig. $2 \mathrm{~B}$ and D), suggesting that HDAC4 expression induces 5-FU resistance in breast cancer. Both the 14-3-3 $\sigma$ and JAK/STAT pathways have recently been shown to be associated with 5-FU resistance in $\mathrm{MCF}-7$ cells $(29,30)$.
We profiled genes that were altered by HDAC4 overexpression as HDAC4 is known to regulate the transcription and stability of other genes and pathways related to acquired resistance through HDAC4 overexpression (Fig. 3A-C). The results of our studies indicated that genes related to the TGF $\beta$ signaling pathway, including SMAD4, SMAD6, BMP6, ID1, TGF $\beta 2$ and TGF $\beta 3$, were most significantly affected by altered HDAC4 expression (Fig. 3D). Among these genes, SMAD4 has been most commonly reported as downregulated in numerous 
types of cancer. Papageorgis et al (14) previously suggested that SMAD4 inactivation induces malignancy in colon cancer and 5-FU chemoresistance, and the results of a study conducted by Shi et al (31) indicated that reductions in SMAD4 expression promoted cell proliferation and invasion in colorectal carcinomas. In addition, advanced gastric cancer displays lower SMAD4 expression than early gastric cancer (32). In breast cancer, Stuelten et al (33) found decreased levels of SMAD4 expression in breast cancer tissue and demonstrated that low SMAD4 expression was correlated with poor prognosis (34). He et al (35) reported that SMAD4 mRNA and protein levels were associated with poor outcome in glioma patients. Of note, our study confirmed the HDAC4-mediated downregulation of SMAD4 in HDAC4OE cells (Fig. 4A and B). Moreover, we verified that SMAD4 mediated 5-FU resistance in MCF-7 cells (Fig. 4C and D). As SMAD4 has been shown to suppress tumor growth by inducing apoptosis in MCF-7 cells (36), HDAC4-mediated regulation of SMAD4 transcriptional expression may be one of the mechanisms underlying 5-FU resistance.

Most genes develop transcriptional regulation through epigenetic modifications, such as methylation and acetylation, without alteration to their DNA sequences. For example, HDAC4 was shown to regulate histone $\mathrm{H} 3$ acetylation in the p21 proximal promoter in colon, ovarian cancer, and osteosarcoma cells $(37,38)$. In addition, Reddy et al (24) suggested that HDAC4 forms a nucleosome remodeling and deacetylating (NuRD) complex in MCF-7 cells and that this complex represses tumor-suppressing gene transcription. In our study, the SMAD4 promoter appeared to have reduced histone $\mathrm{H} 3$ acetylation following HDAC4 overexpression (Fig. 5B), and based on these findings, we suggest that increased deacetylation of the SMAD4 promoter due to HDAC4-mediated negative regulation of SMAD4 gene expression may lead to chemoresistance.

In conclusion, we provide evidence supporting the relevance of HDAC4 expression for 5-FU resistance in breast cancer as well as the molecular basis for HDAC4-mediated gene regulation. We also suggest that HDAC4 regulates SMAD4 expression and modifies histone $\mathrm{H} 3$ in the SMAD4 promoter region.

\section{Acknowledgements}

The present study was supported by the Basic Science Research Program through the National Research Foundation of Korea (NRF), funded by the Ministry of Education, Science and Technology (2010-0007110).

\section{References}

1. Jones PA and Baylin SB: The epigenomics of cancer. Cell 128: 683-692, 2007

2. Ellis L, Atadja PW and Johnstone RW: Epigenetics in cancer: targeting chromatin modifications. Mol Cancer Ther 8: 1409-1420, 2009.

3. Sandoval J and Esteller M: Cancer epigenomics: beyond genomics. Curr Opin Genet Dev 22: 50-55, 2012.

4. Nakagawa M, Oda Y, Eguchi T, et al: Expression profile of class I histone deacetylases in human cancer tissues. Oncol Rep 18 769-774, 2007.

5. Khan O and La Thangue NB: HDAC inhibitors in cancer biology: emerging mechanisms and clinical applications. Immunol Cell Biol 90: 85-94, 2012.
6. Dell'Aversana C, Lepore I and Altucci L: HDAC modulation and cell death in the clinic. Exp Cell Res 318: 1229-1244, 2012.

7. Song B, Wang Y, Xi Y, et al: Mechanism of chemoresistance mediated by miR-140 in human osteosarcoma and colon cancer cells. Oncogene 28: 4065-4074, 2009.

8. Crea F, Nobili S, Paolicchi E, et al: Epigenetics and chemoresistance in colorectal cancer: an opportunity for treatment tailoring and novel therapeutic strategies. Drug Resist Updat 14: 280-296, 2011.

9. Geng H, Harvey CT, Pittsenbarger J, et al: HDAC4 protein regulates HIF1 $\alpha$ protein lysine acetylation and cancer cell response to hypoxia. J Biol Chem 286: 38095-38102, 2011.

10. Tinari N, De Tursi M, Grassadonia A, et al: An epigenetic approach to pancreatic cancer treatment: the prospective role of histone deacetylase inhibitors. Curr Cancer Drug Targets 12: 439-452, 2012

11. Kim MG, Pak JH, Choi WH, Park JY, Nam JH and Kim JH: The relationship between cisplatin resistance and histone deacetylase isoform overexpression in epithelial ovarian cancer cell lines. J Gynecol Oncol 23: 182-189, 2012.

12. Park SY, Jun JA, Jeong KJ, et al: Histone deacetylases 1, 6 and 8 are critical for invasion in breast cancer. Oncol Rep 25: 1677-1681, 2011.

13. Stronach EA, Alfraidi A, Rama N, et al: HDAC4-regulated STAT1 activation mediates platinum resistance in ovarian cancer. Cancer Res 71: 4412-4422, 2011.

14. Papageorgis P, Cheng K, Ozturk S, et al: Smad4 inactivation promotes malignancy and drug resistance of colon cancer. Cancer Res 71: 998-1008, 2011.

15. Takagi Y, Kohmura H, Futamura M, et al: Somatic alterations of the DPC4 gene in human colorectal cancers in vivo. Gastroenterology 111: 1369-1372, 1996.

16. Wang LH, Kim SH, Lee JH, et al: Inactivation of SMAD4 tumor suppressor gene during gastric carcinoma progression. Clin Cancer Res 13: 102-110, 2007.

17. Hahn SA, Schutte M, Hoque AT, et al: DPC4, a candidate tumor suppressor gene at human chromosome 18q21.1. Science 271: 350-353, 1996.

18. Schutte M, Hruban RH, Hedrick L, et al: DPC4 gene in various tumor types. Cancer Res 56: 2527-2530, 1996.

19. Nagatake M, Takagi Y, Osada H, et al: Somatic in vivo alterations of the DPC4 gene at 18q21 in human lung cancers. Cancer Res 56: 2718-2720, 1996.

20. Aitchison AA, Veerakumarasivam A, Vias M, et al: Promoter methylation correlates with reduced Smad4 expression in advanced prostate cancer. Prostate 68: 661-674, 2008.

21. Han S, Lu J, Zhang Y, et al: Recruitment of histone deacetylase 4 by transcription factors represses interleukin-5 transcription. Biochemical J 400: 439-448, 2006.

22. Wang X, Feng Y, Xu L, et al: YY1 restrained cell senescence through repressing the transcription of p16. Biochim Biophys Acta 1783: 1876-1883, 2008.

23. Ren G, Zhang G, Dong Z, et al: Recruitment of HDAC4 by transcription factor YY1 represses HOXB13 to affect cell growth in AR-negative prostate cancers. Int J Biochem Cell Biol 41: 1094-1101, 2009.

24. Reddy SD, Pakala SB, Molli PR, et al: Metastasis-associated protein 1/histone deacetylase 4-nucleosome remodeling and deacetylase complex regulates phosphatase and tensin homolog gene expression and function. J Biol Chem 287: 27843-27850, 2012.

25. Fraga MF, Ballestar E, Villar-Garea A, et al: Loss of acetylation at Lys16 and trimethylation at Lys20 of histone $\mathrm{H} 4$ is a common hallmark of human cancer. Nat Genet 37: 391-400, 2005.

26. Elsheikh SE, Green AR, Rakha EA, et al: Global histone modifications in breast cancer correlate with tumor phenotypes, prognostic factors, and patient outcome. Cancer Res 69: 3802-3809, 2009.

27. Seligson DB, Horvath S, Shi T, et al: Global histone modification patterns predict risk of prostate cancer recurrence. Nature 435: 1262-1266, 2005

28. Barlési F, Giaccone G, Gallegos-Ruiz MI, et al: Global histone modifications predict prognosis of resected non small-cell lung cancer. J Clin Oncol 25: 4358-4364, 2007.

29. Uluer ET, Aydemir I, Inan S, Ozbilgin K and Vatansever HS: Effects of 5-fluorouracil and gemcitabine on a breast cancer cell line (MCF-7) via the JAK/STAT pathway. Acta Histochem 114: 641-646, 2012.

30. Zheng G, Xiong Y, Yi S, et al: $14-3-3 \sigma$ regulation by $\mathrm{p} 53$ mediates a chemotherapy response to 5-fluorouracil in MCF-7 breast cancer cells via Akt inactivation. FEBS Lett 586: 163-168, 2012. 
31. Shi Q, Zhong YS, Yao LQ, et al: Down-regulation of Smad4 enhances proliferation and invasion of colorectal carcinoma HCT116 cells and up-regulates Id2. Mol Med Rep 5: 89-95, 2012.

32. Kim JY, Park DY, Kim GH, et al: Smad4 expression in gastric adenoma and adenocarcinoma: frequent loss of expression in diffuse type of gastric adenocarcinoma. Histol Histopathol 20: 543-549, 2005 .

33. Stuelten CH, Buck MB, Dippon J, Roberts AB, Fritz P and Knabbe C: Smad4-expression is decreased in breast cancer tissues: a retrospective study. BMC cancer 6: 25, 2006.

34. de Kruijf EM, Dekker TJ, Hawinkels LJ, et al: The prognostic role of TGF- $\beta$ signaling pathway in breast cancer patients. Ann Oncol 24: 384-390, 2013.
35. He SM, Zhao ZW, Wang Y, et al: Reduced expression of SMAD4 in gliomas correlates with progression and survival of patients. J Exp Clin Cancer Res 30: 70, 2011.

36. Li Q, Wu L, Oelschlager DK, et al: Smad4 inhibits tumor growth by inducing apoptosis in estrogen receptor-alpha-positive breast cancer cells. J Biol Chem 280: 27022-27028, 2005.

37. Wilson AJ, Byun DS, Nasser S, et al: HDAC4 promotes growth of colon cancer cells via repression of p21. Mol Biol Cell 19: 4062-4075, 2008.

38. Mottet D, Pirotte S, Lamour V, et al: HDAC4 represses p21 (WAF1/Cip1) expression in human cancer cells through a Sp1-dependent, p53-independent mechanism. Oncogene 28: 243-256, 2009. 